\title{
ESTUDIO COMPARATIVO DE LA PRODUCCIÓN DE MIEL CON UNA Y TRES REINAS POR COLMENA EN LA ZONA DE NUEVA IMPERIAL, CHILE
}

\author{
COMPARATIVE STUDY OF HONEY BEE PRODUCTION WITH ONE AND \\ THREE QUEENS BY BEEHIVE IN NUEVA IMPERIAL, IX REGION, CHILE
}

\author{
Ramón Rebolledo R. ${ }^{1}$; Carmen Gloria Guiñez V. ${ }^{1}$; Ximena Araneda D. ${ }^{2}$; Alfonso Aguilera P. ${ }^{1}$
}

\section{RESUMEN}

En la Región de La Araucanía se han realizado ensayos donde se comparó la producción de miel de colmenas doble reina y colmenas de una sola reina, con resultados alentadores. Esto motivó la realización de este estudio en la zona de Nueva Imperial, el cual comparó la producción de miel de sistemas de triple reina y sistemas convencionales con una reina por colmena. Para la realización de este estudio se utilizaron 12 colmenas, de las cuales nueve se agruparon para formar los sistemas triple reina, y las tres restantes con una reina fueron utilizadas como testigo. La investigación comenzó el 4 de noviembre del 2004, con visitas semanales, para controlar los factores que pudieran incidir en la producción de miel. La cosecha se realizó el 11 de enero de 2005, alcanzándose producciones promedio de $85 \mathrm{~kg}$ de miel para los sistemas triple reina, lo cual fue significativamente mayor a la producción lograda por los sistemas con una sola reina que alcanzaron 19,17 kg de miel promedio.

Palabras clave: Triple reina, producción de miel.

\begin{abstract}
In the Region of La Araucania tests have been made where the production of honey of double queen beehives was compared to single queen beehives, with encouraging results. This motivated the accomplishment of this study in the zone of Nueva Imperial, which compared the conventional production of honey of triple queen systems and systems with one queen by beehive. For the accomplishment of this study 12 beehives were used, nine were grouped to form the triple queen systems and the remaining three with one queen were used as control. The research began on November $4^{\text {th }} 2004$, with weekly visits to control the factors that could affect the honey productions. The crop was made on January $11^{\text {th }} 2005$, reaching a honey production of $85 \mathrm{~kg}$ average for triple queens systems, which was significantly greater to the production obtained by the systems with a single queen which reached $19,17 \mathrm{~kg}$ of honey average.
\end{abstract}

Key words: Triple queens, honey production.

\section{INTRODUCCIÓN}

Moeller (1987) define el establecimiento de una colmena como un sistema que se basa en la coexistencia armoniosa de dos o más reinas en una sola unidad. Gutiérrez y Rebolledo (2000) y García (2002) señalan que este sistema tiene como objetivo tener a estas reinas poniendo independientemente en sus respectivos nidos de cría, separados durante la precosecha y cosecha.
Los sistemas plurirreinas se han ideado con el fin de obtener altos rendimientos de miel por colmena, teniendo colmenas con grandes poblaciones durante el flujo de miel (Banker, 1975). Según Garau (1990), la razón de tener una colmena plurirreina es que el mismo número de abejas pecoreadoras en este tipo recoge más néctar que el mismo número de pecoreadoras en colmenas separadas. Con respecto a esto Villarroel et al. (1998), Werthein (1995) y Gutiérrez y Rebolledo (2000) coinciden en que no

$\begin{array}{ll}1 & \text { Universidad de la Frontera. Facultad de Ciencias Agropecuarias y Forestales. Temuco, Chile. } \\ 2 & \text { Universidad Católica de Temuco. Facultad de Recursos Naturales. Temuco, Chile. }\end{array}$

Fecha de Recepción: 05 Octubre 2006

Fecha de Aceptación: 04 Enero 2007 
es lo mismo tener dos colmenas separadas, que una sola con dos reinas.

La mayor producción de miel de un sistema plurirreina es debido a un mayor número de pecoreadoreas (Philippe, 1990). Al respecto, Roma (1981) señala que la asociación de dos más colonias provoca un aumento del calor al interior de la colmena, aumentando con ello la actividad de las obreras, y por ende la productividad, la cual ha sido denominada por este autor como emulación del trabajo. Otro hecho importante según el mismo autor se debe al intercambio de olores (feromonas) entre las colmenas, produciendo de esta manera una emulación de la postura con una suerte de competencia entre las reinas, teniendo como resultado una mayor postura.

La fusión de dos o más colmenas exige cuidados, para evitar que las abejas de las familias a unir luchen entre ellas (Schopflocher, 1986). Esto se debe a que cada colonia tiene su olor característico, que son las feromonas, y que debe ser uno solo en un sistema plurirreina (Crane, 1990; López y De López, 1983). En consecuencia, para establecer un sistema plurirreina es necesario homogeneizar las feromonas producidas por las reinas y obreras que formarán parte de él, logrando así que exista un olor común en toda la colmena y las abejas se sientan parte de una misma unidad (García, 2002; Philippe, 1990).

Existen variadas técnicas para el establecimiento de colmenas plurirreinas, entre los que destacan dos grandes tipos: sistemas de gemelado vertical y horizontal (Philippe, 1990).

En las colmenas plurirreinas de disposición horizontal se encuentra la colmena Onuba de Bravo y Suárez para dos y tres reinas; la colmena Romero; Dunham, Farrar y Perla (Roma, 1981, Garau, 1990). El sistema de gemelado horizontal o yuxtapuesto consiste en colocar dos colmenas en un mueble único, a un mismo nivel, separadas en forma transitoria o permanente por un excluidor de reinas (Figura 5).

El sistema de gemelado vertical consiste en colocar las cámaras de cría una sobre la otra separadas por una rejilla excluidora (Philippe, 1990). Según Garau (1990) y Roma (1981), el sistema más conocido corresponde a la colmena rascacielos.

Respecto a la productividad que este sistema puede alcanzar, Moeller (1987) reporta que en Minnesota (USA) los promedios de miel alcanzados por este sistema llegaron a $127 \mathrm{~kg}$ por colmena de dos reinas y $53 \mathrm{~kg}$ para las colmenas con una sola reina. Hooper (1990) señala producciones en Argentina de más de $150 \mathrm{~kg}$ por colmenas. Experiencias realizadas por la Universidad Autónoma de México señalan que los sistemas plurirreinas producen un $101 \%$ (Promedio de 52,3 kg) más miel que el sistema tradicional de una sola reina $(26,4 \mathrm{~kg}$ de miel) (Gris et al., 2004).

En Chile son pocas las experiencias realizadas con sistemas plurirreinas, a excepción de Susaeta (1968) y González (1979), quienes trabajaron con sistema de doble reina, concluyendo que estos sistemas son más productivos que los de una sola reina, ya que se logran colmenas más vigorosas y con mayor población de pecoreadoras.

En la Región de La Araucanía Villarroel et al. (1998) compararon la producción de miel con una y dos reinas por colmena (gemelado vertical), donde obtuvieron una media de $36 \mathrm{~kg}$ en el sistema doble reina contra $14 \mathrm{~kg}$ en las colmenas de una sola reina. Otro estudio comparativo fue el de Gutiérrez y Rebolledo (2000) en la misma región, quienes compararon la producción de miel en dos sistemas de doble reina (gemelado horizontal y vertical) versus el sistema tradicional (una reina por colmena), obteniendo una producción promedio de $45 \mathrm{~kg}$ para el sistema de gemelado horizontal, $28 \mathrm{~kg}$ en el sistema de gemelado vertical y $8 \mathrm{~kg}$ promedio en el sistema tradicional concluyendo que el sistema gemelado horizontal es mejor que el gemelado vertical.

Por lo anterior, el objetivo planteado en esta investigación fue comparar la producción de miel de un sistema convencional de una reina por colmena versus un sistema de triple reina horizontal en la zona de Nueva Imperial.

\section{MATERIALES Y MÉTODOS}

Las abejas utilizadas en el ensayo correspondieron a la raza ítalo-canadiense, provenientes de un apiario particular, de propiedad de la empresa Araneda e Hija Ltda. Este apiario es manejado en forma tradicional, similar a lo realizado por la mayoría de los apicultores de la región de La Araucanía. La investigación de campo se desarrolló entre las ciudades Imperial-Carahue, específicamente a 9 $\mathrm{km}$ de Carahue, en el sector Lolocura, cuyas coordenadas georreferenciadas mediante GPS marca Garlin modelo III son HUSO 180666767, UTM 5714087, a 10 m.s.n.m. 
El apiario estaba distribuido linealmente, orientado de Oeste a Este, distribución que no fue modificada. Las piqueras de las colmenas tenían una orientación $160^{\circ}$ Sureste. El lugar seleccionado presentaba floración de pradera natural, algunas plantaciones, árboles frutales y bosque nativo. La zona de Nueva Imperial corresponde al secano, en el sector poniente de la Región de La Araucanía (Romero y Demanet, 1988). La zona seleccionada para la actividad experimental está clasificada como Macroárea II por Rouanet (1982).

Para el estudio se seleccionaron 12 colmenas tipo Langstroth, de dos cuerpos cada una. El experimento se llevó a cabo utilizando abejas que salen del invierno, tal como las mantiene el apicultor en la zona, con el objeto de no llevar el trabajo a un plano que no correspondiese a la realidad. Se realizó una visita el 29 de septiembre del 2004, con la finalidad de hacer un reconocimiento del apiario en el cual se establecería el experimento.

La segunda visita, realizada el 13 de octubre del 2004, permitió confeccionar un bosquejo del apiario y un reconocimiento de especies vegetales presentes en el lugar. El día 28 de octubre del 2004 se realizó una revisión del colmenar, con el fin de seleccionar las doce colmenas de características similares para ser utilizadas en el experimento. Se buscó que éstas estuvieran en las mejores condiciones sanitarias, similar cantidad de abejas, cuadros de postura y alimento.

Es importante mencionar que las condiciones climáticas imperantes durante la primavera atrasaron alrededor de 20 días el comienzo del experimento, ya que las lluvias y las bajas temperaturas del mes de octubre no permitieron realizar las labores en las fechas preestablecidas.

El experimento comenzó el jueves 4 de noviembre de 2004. Para la instalación del sistema triple reina se tomaron nueve colmenas de dos cuerpos tipo Langtroth, dejando tres como testigo, agrupadas horizontalmente de tres en tres, al mismo nivel, una junto a la otra, con las piqueras de las orillas orientadas $160^{\circ}$ Sureste, mientras que la piquera central fue orientada en el sentido contrario (Figuras 1, 2 y 3). El cambio de disposición de las piqueras se realizó para evitar que ocurriera deriva entre las colmenas que formaron parte del sistema. El fenómeno de deriva es definido por Prost (1985) como el cambio de colonia de las pecoreadoras a pesar de la señalización de su domicilio. Esta se puede manifestar en colonias dispuestas en filas

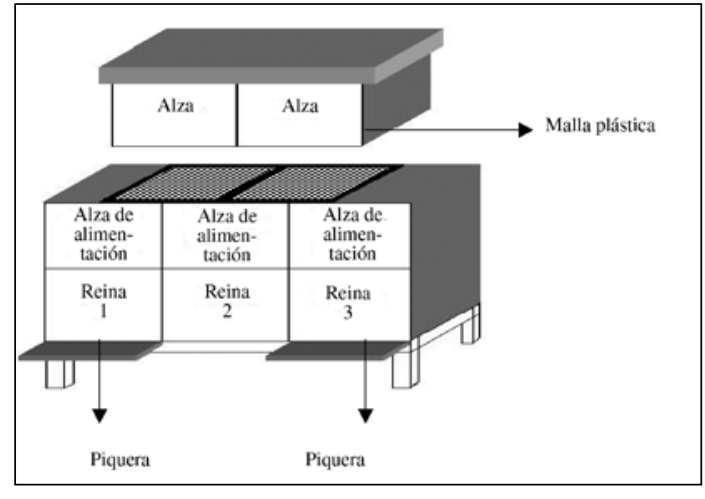

Figura 1. Esquema sistema triple reina mostrando la malla de homogeneización de olores.

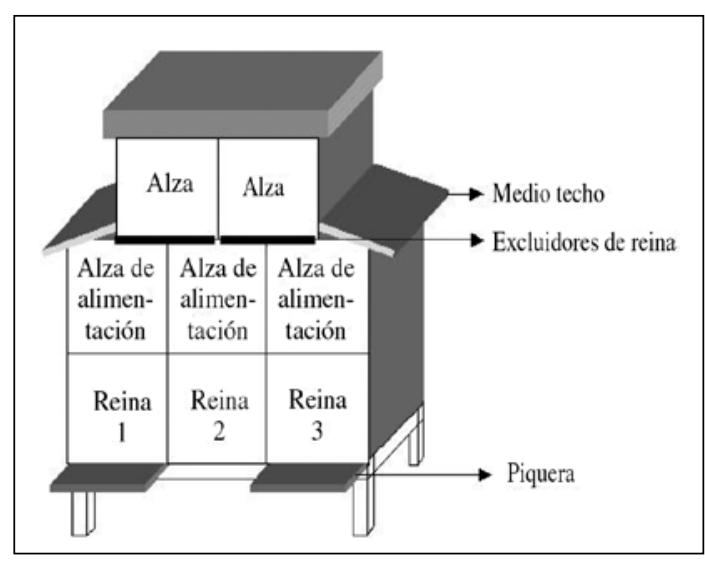

Figura 2. Vista anterior del sistema triple reina.

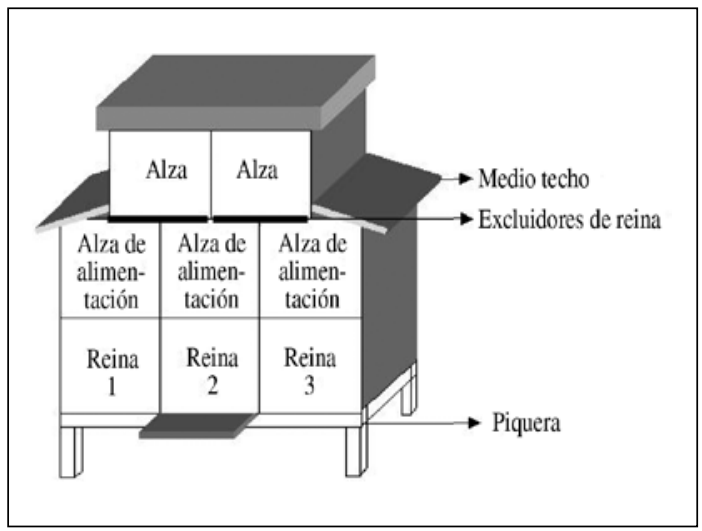

Figura 3. Vista posterior del sistema triple reina.

paralelas con las piqueras orientadas en la misma dirección. Por ejemplo, frente a las flores a cosechar, las colmenas de la primera fila captan pecoreadoras ajenas a la vuelta del campo, o las colmenas situadas 
en los extremos de las filas recogen pecoreadoras de colmenas más centrales.

Entre las tres colmenas se montó una malla de plástico (Figura 1), y sobre éstas dos alzas con marcos con cera estampada. La función de esta malla es suprimir temporalmente el paso de las abejas hacia las alzas recién puestas y facilitar que se mezclen los olores característicos de cada reina y obreras (feromonas), de manera que al final existiera un olor común en toda la colmena, evitando con esto que ocurrieran posteriores peleas y muerte entre las abejas al retirar la malla y ser reemplazada por la rejilla excluidora de reinas. Así se logró que las tres colonias pudieran funcionar más adelante como una sola unidad. La ubicación de esta malla en el sistema triple reina se puede observar en la figura 1.

Se confeccionaron, además, seis medios techos y seis medios entretechos para proteger los espacios laterales que quedaron descubiertos.

Pasado seis días (10 de noviembre de 2004), se retiraron las mallas plásticas, las que fueron reemplazadas por excluidores de reinas, lo que permitió el libre tránsito de las abejas desde las tres cámaras hacia las alzas superiores. En este momento comenzó a funcionar el experimento como tal. La distribución de las alzas, piqueras y rejillas excluidoras se puede apreciar en las figuras 2 y 3 .

Para el establecimiento de las colmenas testigos fueron seleccionadas tres colmenas tipo Langtroth de dos cuerpos, una a cada lado del sistema triple reina, para que los resultados fueran lo más representativos posibles. Estas se manejaron de manera tradicional, similar al procedimiento realizado con el sistema triple reina. Se les agregaron alzas en la medida que se fueron necesitando, y marcos con cera estampada en las cámaras de cría para dar lugar a la postura de la reina. En la figura 4 se puede apreciar el sistema triple reina, y a su lado la colmena testigo. En la figura 5 se observan las cámaras de cría con sus alzas de alimentación, y sobre éstas los excluidores de reina.

La distribución de las colmenas en el apiario donde se estableció el experimento se muestra en la figura 6. Este estaba formado por tres hileras de colmenas, en cada una de las cuales se ubicó un sistema triple reina y a su lado la colmena testigo (Figura 4).

Es importante mencionar que tanto a las colmenas testigos como a las triple reina se les agregaron alzas con marcos con cera estampada y no estirada, lo que obligó a las abejas a un mayor trabajo, ya que deben estirar la cera antes de utilizarlas.

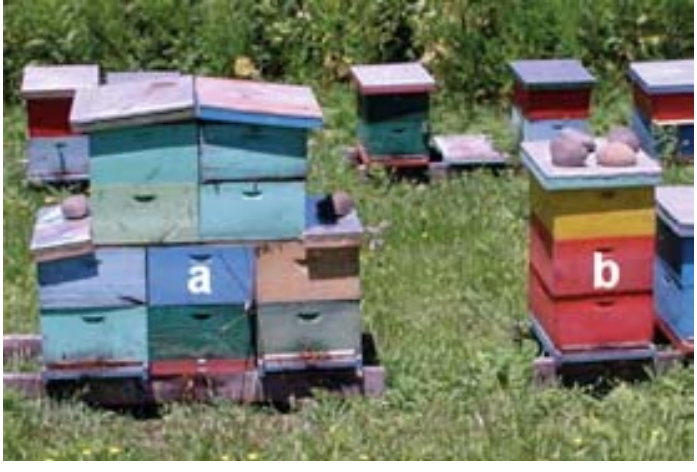

Figura 4. Sistema triple reina y su respectivo testigo $(a=$ Sistema triple reina; $\mathrm{b}=$ Colmena testigo).

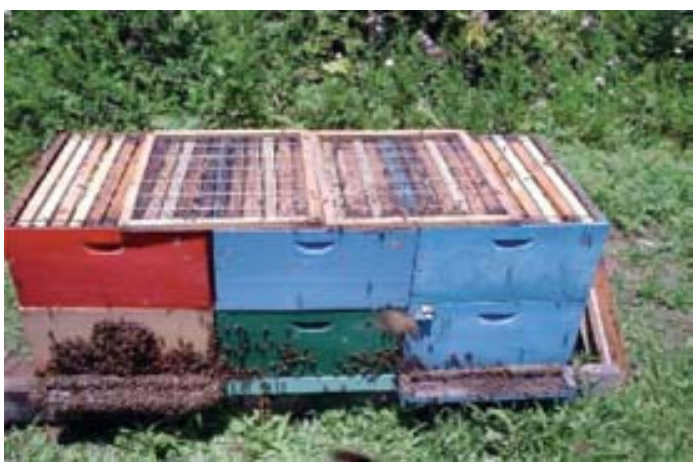

Figura 5. Alzas y rejilla excluidora de reina en el sistema triple reina.

El manejo realizado a las colmenas consistió en una revisión periódica, una vez por semana, desde el montaje del sistema, el 4 de noviembre de 2004, hasta la fecha de la cosecha. Las colmenas fueron revisadas con la finalidad de controlar aquellos factores que pudieran incidir en la producción de miel. Las labores realizadas en los sistemas triple reina y en las colmenas testigo fueron las mismas, para no llevar el experimento a condiciones que no corresponden a la realidad. Estas labores consistieron principalmente en:

- Destrucción de las celdillas reales que se encontraban en los marcos. Esto se realizó tanto en los sistemas triple reina como en los sistemas convencionales o testigos.

- Se facilitó espacio mediante la colocación de alzas a medida que lo fueron necesitando. La primera alza fue puesta cuando se estableció el sistema. Ésta fue la misma utilizada para la homogeneización de olores. La segunda alza 
común fue instalada el 18 de noviembre, o sea, ocho días después que comenzó a funcionar el sistema triple reina como tal.

- Facilitar espacio a la reina para su postura, realizando un traslado de marcos con cría y abejas a la segunda alza común, para incentivar el ascenso de las abejas desde las cámaras inferiores y estimular la producción de miel. En la cámara de cría y primera alza individual de cada colmena, los marcos retirados fueron reemplazados con marcos con cera estampada, para dar más espacio a la reina para su postura.

La cosecha se realizó el 11 de enero del 2005 , cuando se verificó que más del $75 \%$ de las celdillas estaban operculadas. En los marcos no operculados, si al sacudirlos la miel no gotea, es señal de que está madura y puede ser cosechada.

Los marcos fueron puestos en alzas vacías y éstas rotuladas, para posteriormente trasladarlas a la sala de cosecha, ubicada en la ciudad de Nueva Imperial. Allí las alzas fueron pesadas con todos los marcos en su interior, luego los marcos fueron puestos en la máquina desoperculadora, centrifugados y la miel puesta en tambores. Los marcos, luego de ser centrifugados, se pusieron nuevamente en las alzas, y éstas pesadas. Se obtuvo el rendimiento de miel por diferencia de peso. La actividad de cosecha se realizó una sola vez y no se practicó transhumancia en ninguno de los sistemas. Aunque las colmenas pudieran eventualmente ser cosechadas una segunda vez, esto no se realizó por necesidades del dueño del apiario. Además, una segunda cosecha muy tarde en la temporada expone a las colonias a falta de alimento para el invierno por el término de la floración y riesgo de pillaje.

El diseño experimental utilizado fue completamente al azar con dos tratamientos, colonias simples con una reina por colmena, y colonias triple reina con tres reinas por colmena. Cada tratamiento contó con tres repeticiones. Para el análisis de los datos se recurrió a la estadística no paramétrica, empleándose la prueba U de Mann-Whitney (Siegel, 1983).

\section{RESULTADOS Y DISCUSIÓN}

Los resultados generales de la producción de miel alcanzados por los sistemas testigos y triple reina están representados en la figura 6. En ésta se destaca una clara superioridad en el rendimiento de las colmenas triple reina que alcanzaron una producción promedio de $85 \mathrm{~kg}$ de miel, comparado con los $19 \mathrm{~kg}$ de miel obtenidos por las colmenas testigo con una diferencia de $66 \mathrm{~kg}$ entre ambos sistemas, lo que fue estadísticamente significativo. Lo anterior significó un $82 \%$ del total obtenido en la cosecha del sistema triple reina contra el 18\% restante del sistema de producción tradicional. Estos resultados coinciden con Susaeta (1968), González (1979), Villarroel et al. (1988), Gutiérrez y Rebolledo (2000) y Gris et al. (2004) quienes encontraron que el sistema plurirreina produce más miel por unidad, aunque ellos trabajaron con sistemas de doble reina.

La figura 7 muestra las producciones individuales de cada uno de los sistemas. En ésta se

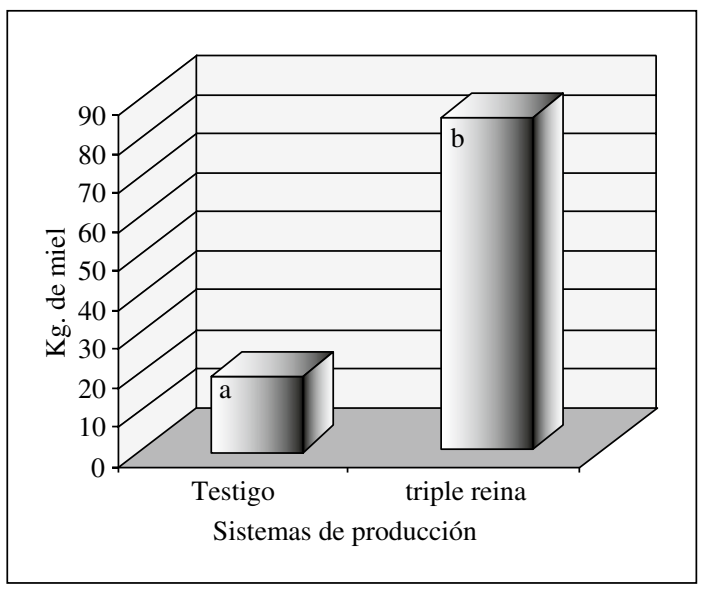

Figura 6. Producción promedio con una y tres reinas por colmenas.

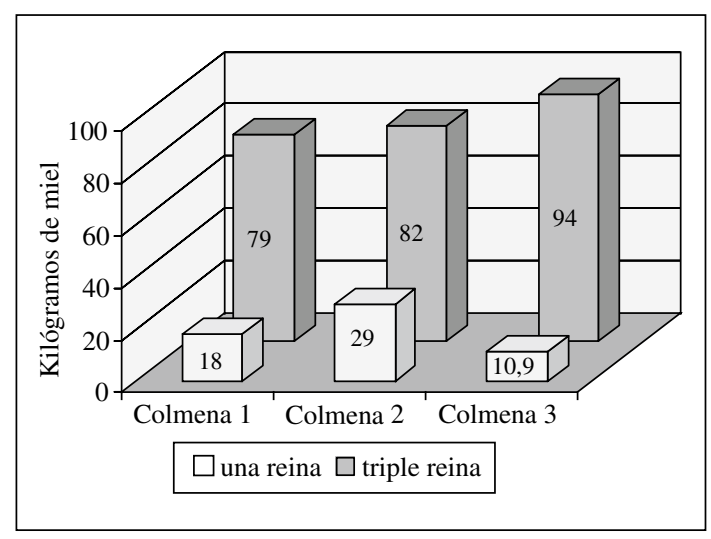

Figura 7. Producción de miel con una y tres reinas por colmenas. 
puede observar que en las colmenas de una sola reina, fue la número dos la que logró la mayor producción, mientras que la colmena tres tuvo la menor. En la misma figura se observa que en el caso de las colmenas triple reina fue la número tres la que alcanzó los mayores valores de producción con $94 \mathrm{~kg}$ de miel, mientras que la colmena uno fue la que obtuvo la menor producción con $79 \mathrm{~kg}$ de miel.

No hubo diferencias en el tiempo de maduración entre ambos sistemas, ya que al momento de la cosecha, tanto las colonias testigo como las triple reina se encontraban en similares condiciones. En los sistemas triple reina se observó una mayor población de abejas, considerablemente superior a los sistemas de reina única. Esto hace suponer una posible producción de núcleos superior al sistema tradicional consiguiendo con ello una mayor rentabilidad por unidad. En este respecto, García (2002) señala como conclusión de varias experiencias realizadas en Argentina, que la producción de núcleos es una alternativa viable con colmenas plurirreinas.

En las diferentes visitas realizadas durante el estudio se comprobó que el tiempo empleado en revisar las colmenas de una sola reina es mucho más breve y menos agotador, debido a que el sistema triple reina presenta mayor número de unidades a mover. Otro aspecto que demandó mucho esfuerzo fue la prevención de la enjambrazón, que resultó mucho más complicado en el sistema triple reina, inclusive cuando se trasladaron panales con huevos

\section{LITERATURA CITADA}

BANKER, R. 1975. Manejo de colonias con dos reinas. En Dadant e Hijos. La colmena y la abeja melífera. Hemisferio Sur. Montevideo. Uruguay. Pp. 523-531.

CRANE, E. 1990. Bees and beekeeping. Comstock Publishing Associates. Ithaca, New York. Pp. 250-253.

GARCÍA, M. 2002. Fundamentos de la producción apícola moderna. Encestando S.R.L. Argentina. 187 p.

GARAU, J. 1990. Curso Superior de Apicultura. Palmas de Mallorca. España. Pp. 236-250.

GONZÁLEZ, M. 1979. Estudio comparativo de la producción de miel entre los sistemas de doble reina y tradicional de reina única en la provincia de Nuble. Tesis para optar al título de Médico Veterinario. Universidad de Concepción. Chillán, Chile. 40 p.

GRIS, A.; E. GUZMÁN; A. CORREA Y A. ZORAYA. 2004. Efecto del uso de dos reinas en la población, peso, producción de miel y rentabilidad de colonias de abejas a las alzas mielarias, es decir, aquellas que estaban sobre las rejillas excluidoras, las abejas estiraron celdillas reales que fue necesario destruir, situación que se remedió agregando panales con cría operculada. A pesar de la revisión periódica, de todas formas una de las unidades del triple reina enjambró, específicamente la colmena número 2. Aun a pesar de ello, esta colmena triple reina logró una producción de 82 kilogramos.

Otra de las dificultades que presentó el sistema triple reina fue ubicar la reina, aspecto importante en este estudio. Por tanto, un requisito esencial para un buen manejo de este sistema es marcar las reinas de la temporada para facilitar la prevención del enjambre. Finalmente, es necesario señalar que el sistema de triple reina requiere necesariamente de entrenamiento para llevarlo a escala comercial.

\section{CONCLUSIÓN}

Del presente trabajo y bajo las condiciones en que se realizó la investigación se concluye que el sistema triple reina produce significativamente más miel que el sistema convencional de una reina por colmena. No existen diferencias en el tiempo de maduración de la miel. El sistema triple reina demandó mayor número de labores que el sistema testigo de una sola reina por colmena y se estima la necesidad de marcar las reinas para el manejo eficaz del sistema.
(Apis mellifera L.) en el antiplano de México. Disponible en http://www.tecnicapecuaria.org.mx/publicaciones/ publicacion04.php?IdPublicacion=437. Consultada el 23 de noviembre 2004.

GUTIÉRREZ, J. Y R. REBOLLEDO. 2000. Comparación de la producción de miel de dos sistemas de doble reina y un sistema tradicional de una reina por colmena. Agro Sur 28(2): 11-14.

HOOPER, J. 1990. Las abejas y la miel. Guía para el apicultor. Editorial El Ateneo. Buenos Aires. Pp. 163-195.

LÓPEZ, M. Y G. DE LÓPEZ. 1983. Tratado sobre las abejas. Ed. Albatros. Buenos Aires. 467 p.

MOELLER, M. 1987. Administración de las colonias para lograr un alto rendimiento de miel. En McGregor. La Apicultura en los Estados Unidos. Limusa. México. Pp. 23-30.

PHILIPPE, J. 1990. Guía del apicultor. Mundi-Prensa. Madrid. España. 376 p. 
PROST, P. 1985. Apicultura. Conocimientos de la abeja. Manejo de la colmena. Mundi-Prensa, Madrid. 573 p.

ROMA, A. 1981. Explotación intensiva del colmenar. Sintes. Barcelona. $355 \mathrm{p}$.

ROMERO, O. Y R. DEMANET. 1988. La pradera en el secano de la IX Región (suelos rojo-arcillosos). En Ruiz, I. Praderas para Chile. Instituto de Investigación Agropecuarias. INIA. Ministerio de Agricultura. Santiago, Chile. pp. 453-466.

ROUANET, J. 1982. Áreas agroecológicas determinantes del sistema de producción agropecuaria. Investigación y Progreso Agropecuario Carillanca. Instituto de Investigación Agropecuaria. Temuco, Chile 1(1): 17-21.

SCHOPFLOCHER, R. 1986. Apicultura lucrativa. Albatros. Buenos Aires. $185 \mathrm{p}$
SIEGEL, S. 1983. Estadística no paramétrica aplicada a las ciencias de la conducta. Ed. Trillas. México. Pp. 143-155.

SUSAETA, M. 1968. Aplicación de un sistema de dos reinas para la polinización de alfalfa y trébol rosado. Investigación y Progreso Agrícola. Instituto de Investigaciones Agropecuarias. Santiago, Chile. 2: 9-12.

VILLARROEL, D.; R. REBOLLEDO Y A. AGUILERA. 1998. Estudio comparativo de la producción de miel con una y dos reinas por colmena en la zona de Nueva Imperial, IX Región, Chile. Agro Sur 26(2): 121-126.

WERTHEIN, I. 1995. El apicultor. El futuro privilegiado de la industria agropecuaria. El Arca Ediciones. Argentina. Pp. 12-15. 